3. Anonymität und Transparenz, Autonomie und Verantwortung in digitalen Öffentlichkeiten 


\section{Namenlos, durch das Netz}

\section{Anonymität und Transparenz in digitalen Kulturen}

\section{Lea Watzinger}

\section{Anonymität und Transparenz als Probleme digitaler Kulturen}

Die gesellschaftlichen Veränderungen durch digitale Kommunikation und ihre Auswirkungen auf kultureller, zwischenmenschlicher, juristischer wie auf politischer Ebene sind umfassend, was grundsätzliche Reflexionen nötig macht: Der vorliegende Aufsatz nimmt daher eine philosophische Perspektive ein, aus der Begriffe, die in der Alltagssprache geläufig sind, in einen breiteren ideengeschichtlichen Kontext gestellt und kritisch reflektiert werden. Im Fokus stehen dabei die Begriffe der Anonymität sowie der Transparenz, die beide Schlüsselbegriffe des digitalen Wandels sind.

Digitale Kommunikation wird häufig mit Anonymität (bzw. Pseudonymität $)^{1}$ in Verbindung gebracht. Ein Blick auf Internetforen oder Kommentarspalten, in denen Nutzende unter Pseudonymen posten und sich hinter diesen ,verstecken', sowie Diskussionen um das sogenannte Dark Net und dessen kriminelles Potential scheinen dies zu bestätigen. ${ }^{2}$ Gleichzeitig wohnt digitaler Kommunikation die Möglichkeit weitreichender Transparenz und Nachverfolgbarkeit inne. So ergibt sich im Rahmen digitaler Kommunikation ein Spannungsfeld zwischen diesem Antagonismus aus Anonymität und Transparenz und daraus resultierenden Verantwortlichkeiten. Die Zuschreibung von persönlichen Verantwortlichkeiten, welche eine Grundlage menschlichen Zusammenlebens ist, gerät ins Wanken, wenn Handlungen und Äußerungen im Internet keinem Individuum zugeordnet werden können. Die Kommunizierenden werden gewissermaßen unsichtbar, sodass es nicht immer und offensichtlich klar ist, wo und wer

1 Die Alltagssprache verwendet beide Begriffe meist synonym, während in der juristischen Debatte eher von Pseudonymität gesprochen wird, selbst wenn in der Alltagsprache häufig Anonymität gemeint ist. Für die Rechtswissenschaften ist die Unterscheidung zwischen Anonymität und Pseudonymität von hoher Relevanz, vgl. hierzu Härting, NJW 2013, 2065. Dabei steht die Bereinigung persönlicher Daten um individuelle Identifikationsmerkmale im Zentrum.

2 Vgl. für eine Einführung z. B. Mey (2018). 
sie sind. Dass es sich beim Internet um einen Raum der Anonymität handele, sei laut Thorsten Thiel jedoch eine „Wahrnehmungstäuschung“. ${ }^{3}$ Im Internet seien wir gar nicht anonymer als in der analogen Welt, es könne lediglich dieser Eindruck entstehen, da es auf der horizontalen Ebene möglich sei, Identitäten zu verschleiern, und da die physische Präsenz wegfalle. Gleichzeitig hinterlässt jede digitale Transaktion Daten und ist damit nachverfolgbar, macht also die Einzelnen transparent. ${ }^{4}$

In diesem Beitrag diskutierte Fragen sind, welche Dimensionen Anonymität und Transparenz vor allem bezogen auf digitale Kulturen annehmen und wie sich diese auf die Verantwortlichkeit von Personen auswirken können. Dazu werden die theoretischen Positionen von Michel Foucault und Hannah Arendt mit aktuellen Überlegungen, wie denen von Thorsten Thiel, zur digitalen Kommunikation verbunden, wobei die Anonymität des Individuums in Handlungs- und Sprechsituationen den einen Fokus darstellt, während die Transparenz der Einzelnen durch Datenpreisgabe im Rahmen der Nutzung digitaler Medien den anderen bildet.

\subsection{Was bedeutet Anonymität?}

Um ein erstes Verständnis von Anonymität zu entwickeln, soll Michel Foucaults einflussreicher Beitrag hierzu herangezogen werden. So ist ein zentraler Aspekt von Anonymität - griechischer Begriff für Namenlosigkeit - die ,Urheber-' bzw. ,Autorschaft ${ }^{6}{ }^{5}$ Von geschriebenen Texten ausgehend bemerkt Foucault zur Nennung bzw. Nicht-Nennung des Autornamens in Texten: „Ein anonymer Text, den man an einer Hauswand liest, wird einen Verfasser haben, aber keinen Autor. Die Funktion Autor ist also charakteristisch für Existenz-, Verbreitungs- und Funktionsweisen bestimmter Diskurse in einer Gesellschaft." 6

Foucault unterscheidet hier zwischen Verfasser und Autor, also zwischen Äußerungen ohne und Äußerungen mit Verantwortungsübernahme. Autorschaft hat für ihn eine attributive, eine zuweisende Funktion. Der Autor eines Textes übernimmt Verantwortung für das Geschriebene und diese kann ihm entsprechend zuerkannt werden. Namhaftigkeit führt

3 Thiel (2016: S. 17).

4 Vgl. Matzner (2016: S. 248).

5 In dieser Untersuchung wird mehrmals von ,Autorschaft', ,Verfasser ${ }^{\varsigma}$ und ,Urheberschaft' als Termini technici in Anlehnung an Foucault die Rede sein, vgl. Foucault (2000).

6 Foucault (2012: S. 211). 
also gewissermaßen zu Verantwortlichkeit, der Autor kann zur Rechenschaft gezogen werden, der (bloße) Verfasser nicht, so Foucaults Unterscheidung. Letzterer bleibe anonym, also in der Namenlosigkeit, was eine Rückführung des Geschriebenen auf eine Person und damit die Zuschreibung von Verantwortung unmöglich mache. ${ }^{7}$ Mit der Autorschaft als Gegenbegriff zur Anonymität rückt Foucault folglich die Verantwortlichkeit für Geschriebenes in den Blick und leitet daraus eine Verpflichtung ab, etwaige Konsequenzen zu tragen.

Einen auf digitale Kulturen angewandten Begriff von Anonymität entwickelt Thorsten Thiel, der Anonymität anders als Foucault nicht als Nichtgenanntsein und Namenlosigkeit versteht, sondern als „eine $\mathrm{Zu}$ standsbeschreibung in einer intersubjektiven Handlungssituation, in der es unmöglich ist, eine Handlung oder Kommunikation einem Individuum oder Subjekt korrekt zuzuordnen und es über den Kontext hinaus erreichbar/verantwortlich zu machen [...]." 8

Thiel fokussiert also auf die Situationsgebundenheit von Anonymität im intersubjektiven Austausch und die Schwierigkeit, das kommunikative Gegenüber zu identifizieren. Auch hier geht es um die Verbindung einer Handlung mit einer urhebenden Person und damit um die Frage, wer die Verantwortung für diese Handlung übernimmt. Thiel betont, dass Anonymität keine feste und dauerhafte Eigenschaft einer Person sei, sondern unter den Bedingungen einer bestimmten Handlungssituation bestehe. Sie kann - gerade im digitalen Bereich - auch rückwirkend aufgehoben werden, ist also nicht fix und endgültig, sondern stets situativ. Wie anonym jemand in einer Situation ist, hängt also maßgeblich davon ab, wie leicht und durch wen eine Person identifiziert werden kann. ${ }^{9}$

Die beiden bis hierher vorgestellten Positionen von Foucault und Thiel zeigen, dass Anonymität ein mehrdimensionaler Begriff ist. Dabei handelt es sich nicht lediglich um einen beschreibenden Ausdruck, sondern mit ihm gehen potenziell starke normative Wertungen einher, die auf der Annahme fußen, dass Anonymität das Verhalten von Menschen beeinflusst. Einschätzungen und Beobachtungen, wie dieser Einfluss aussieht, driften weit auseinander - anonyme Bedingungen können augenscheinlich äu-

7 Vgl. Foucault (2000: 212 f.).

8 Thiel (2016: S. 10). Thiel unterscheidet außerdem zwischen Anonymität, die durch ,Verschleierung' hergestellt wird und Anonymität, die durch das Aufgehen in einer Masse entsteht. Das Aufgehen in einer Masse mache aus vielen Individuen eines und sie damit ununterscheidbar. Damit verliere sich, so Thiel, die Zuordenbarkeit von Verantwortung. Vgl. ebd. (S. 11).

9 Vgl. Matzner (2016: S. 249). 
ßerst verschiedene Verhaltensweisen hervorbringen, wie Thiel weiter ausführt. Er spricht von ,Aufrichtigkeit‘ oder ,Enthemmung', welche (das Gefühl von) Anonymität in Menschen auslöse:

Die eine These ist die ,Enthemmungsthese', der zufolge eine durch Anonymität erzeugte Verantwortungslosigkeit einen Vertrauens- und Rationalitätsverlust mit sich bringt; dem entgegengestellt ist die ,Aufrichtigkeitsthese', nach der davon auszugehen ist, dass die Entbundenheit von Zuschreibungs- und damit Machtrelationen Authentizität und Unparteilichkeit produziert. ${ }^{10}$

Individuelle Anonymität hat laut diesem Zitat also prinzipiell das Potential, Freiheit und damit demokratische und gesellschaftliche Teilhabe zu ermöglichen oder zu vereinfachen, was in digitalen Kommunikationskontexten besonders virulent wird. So profitieren beispielsweise Personen, die in autoritären Regimen leben und diese kritisieren, davon, diesen gegenüber anonym zu sein. Menschen, die aus dem gesellschaftlichen Mainstream herausfallen oder in einem restriktiven Umfeld leben, profitieren möglicherweise davon, sich in einem Rahmen der Anonymität auszuprobieren und Gleichgesinnte zu suchen; sie erhalten die Möglichkeit, im Schutze der Namenlosigkeit verschiedene Rollen zu erfahren und auszuleben. Anonymität ermöglicht nach so einem Verständnis - online wie offline - die Diversität von Rollen in unterschiedlichen Lebensbereichen und kann zu einer erhöhten Solidarität mit Fremden führen, wo diese unter Klarnamen vielleicht schwerfiele, etwa wenn es um sensible Themen geht. ${ }^{11}$ Andererseits lässt sich jedoch auch das Gegenteil beobachten, wie Thiel bemerkt. Dies ist dann der Fall, wenn das Gefühl der Anonymität bei Individuen gerade nicht die Aufrichtigkeit stärkt, sondern Verantwortungslosigkeit erzeugt. Der Mantel der Namenlosigkeit kann entsprechend Enthemmung bewirken, da nicht nur das Bewusstsein für die Anderen als Personen, sondern auch als Menschen verloren geht. ${ }^{12}$ Anonymität kann somit, wie Thiel verdeutlicht, entweder Bedingung für Freiheit, Demokratie und Öffentlichkeit sein oder diese gefährden. Pauschale Bewertungen von Anonymität im Netz sind daher kaum möglich. ${ }^{13}$

10 Thiel (2016: S. 13).

11 Vgl. Helm (2017).

12 Vgl. hierzu Lück (2013).

13 Vgl. Thiel (2016: S. 19). 


\subsection{Was bedeutet Transparenz?}

Transparenz stellt einen Schlüsselbegriff des beginnenden 21. Jahrhunderts dar, ist jedoch in der philosophischen Ideengeschichte weniger verankert als der Begriff der Anonymität. ,Transparent' bedeutet dabei zunächst einmal durchsichtig, durchscheinend und entstammt dem Feld der Optik. ${ }^{14}$ Inzwischen hat sich der Begriff jedoch aus diesem Anwendungsfeld gelöst und bezieht sich - in einem übertragenen Sinn - auf Nicht-Gegenständliches. Die gegenwärtige moralische Bedeutung von Transparenz resultiert aus dem metaphorischen Wortgebrauch im Bereich von Staat und Gesellschaft: So konnte die Rede von Transparenz die Grenzen der Optik überwinden und sich breitere Anwendungsgebiete im Kontext von Wirtschaft, Politik und Recht erschließen. ${ }^{15}$ Dabei enthält der Transparenzbegriff eine demokratietheoretische sowie eine individuumsbezogene Dimension, weswegen zwischen einer Transparenz des Staates und einer Transparenz des Individuums zu unterscheiden ist. Transparenz als demokratietheoretischer Begriff bezogen auf den Staat versteht diese als ein Mittel gegen Korruption und für Demokratie. Transparenz wird in einem solchen Kontext gleichgesetzt mit Information, worauf (scheinbar) ein Recht besteht: Strukturen sollen transparent sein, also einsehbar, sichtbar, nachvollziehbar. Verantwortlichkeiten im Bereich politischer Vorgänge sollen durch die Herstellung von Transparenz nachverfolgbar und zugänglich werden.

Für den Zusammenhang mit Anonymität und individueller Verantwortung im Internet ist jedoch die zweite Bezugsrichtung von Transparenz auf das Individuum zentral. Im Rahmen einer umfassenden Digitalisierung scheint sich ein ,Ende der Informationskontrolle ${ }^{16}$ anzukündigen, und damit eine weitreichende digitale Transparenz des Individuums. Im Zusammenhang mit diesem ist zwar meist weniger die Rede vom ,transparenten“

14 Vgl. Digitales Wörterbuch der deutschen Sprache (o. J.).

15 Dies bemerkt Armando Menéndez-Viso in seiner philosophischen Reflexion der Widersprüchlichkeiten, die der moralischen Transparenzmetapher innewohnen: "[T]he only non-poetic mentions of transparency could be found in texts about the nature of light, the properties of lens and crystals, the anatomy of the eye, or luminescent fishes and insects-making direct use of the word, to denote precisely a property of physical objects in relation with light. The current, moral meaning of the term comes about just as it starts to be used in a metaphoric fashion by social scientists (and not only by poets), thus trespassing the borders of optics and reaching the ampler territories of economics, sociology, politics and law." Menéndez-Viso (2009: S. 157).

16 Vgl. Hagendorff (2017). 
als vom ,gläsernen` Menschen, doch können beide Ausdrücke weitgehend synonym verstanden werden: Beim Sprachbild des, gläsernen Menschen handelt es sich - wie beim Transparenzbegriff selbst auch - um einen mehrfach metaphorischen Ausdruck, der einerseits aus dem Bereich der Medizin kommt und sich andererseits im Kontext digitaler Welten auf einen abstrahierten Begriff von Sichtbarkeit bezieht. Der, gläserne Mensch` ist semantisch eng verbunden mit, Überwachung' und einer mit dieser einhergehenden Bedrohung. Die Sichtbarkeit, auf die sich der Ausdruck bezieht, ist eine abstrahierte und metaphorische, denn es sind Daten, die ein Individuum nachverfolgbar machen. ${ }^{17}$

So kann der Transparenzbegriff als Schlüsselbegriff digitaler Kulturen verstanden werden, der sich auf das Individuum bezieht und dieses einer normativen Metapher der Sichtbarkeit unterwirft. Die Transparenz des Individuums ist dabei mit verschiedenen Annahmen und normativen Herausforderungen verbunden. Als digital transparentes Individuum wird dieses nachverfolgbar und ,trackbar', was dessen Autonomie und Privatheit einschränkt. Damit steht ,Transparenz' bezogen auf das Individuum im Gegensatz zur ,Privatheit‘, aber auch zur ,Anonymität‘.

Der Transparenzbegriff gelangt also von der Physik in einem übertragenen Sinn in die Sozial- und Geisteswissenschaften. Wie schon hier deutlich wurde, handelt es sich auch beim Transparenzbegriff - wie beim Begriff der Anonymität - um einen deutlich normativen und metaphorischen Ausdruck. ,Transparenz' verschlagwortet die digitale Verschiebung der Grenzen von Öffentlichkeit, Geheimnis und Privatheit und changiert dabei stets zwischen Beschreibung und normativem Anspruch. Transparenz ist eng verbunden mit einer letztlich bis in die Antike reichenden metaphorischen Verbindung von Licht und Sehen mit Erkenntnis und Moral, und zwar sowohl auf etymologischer als auch auf metaphorischer Ebene. ${ }^{18}$ Mit der Aufklärung wird diese Metaphorik in Abgrenzung zum ,dunklen Mittelalter' reaktiviert, wie Bernhard Wegener darstellt: „Das helle Licht der Vernunft, das Tageslicht der öffentlichen Auseinandersetzung, der freien Aussprache in privaten wie in öffentlichen Angelegenheiten avancierte im Laufe dieser Auseinandersetzungen zum Wert an sich. “"19

17 Vgl. Weidacher (2019). Der, gläserne‘ wie auch der ,transparente‘ Mensch meinen im vorliegenden Aufsatz also stets das verdatete und in diesem metaphorischen Sinne transparente Individuum.

18 Seit der Antike, prominent in Platons Höhlen- und Sonnengleichnis expliziert, sind ,Sehen' und Licht mit der Erkenntnis des Wahren und der Ideen verbunden. Das Wort ,Idee‘ (gr. i $\delta \varepsilon \varepsilon \alpha$ ) leitet sich entsprechend von ,sehen` (gr. i $\delta \varepsilon \tilde{i v}$ ) her. 
Es komme dabei zu einer Verherrlichung von Licht, Wahrhaftigkeit sowie Ehrlichkeit und zu einer Abwertung von Verbergen, Dunkelheit und Geheimnis, während letzteres moralisch diskreditiert werde. ${ }^{20}$ Sichtbarkeit und Licht wurden so zu Metaphern von Erkenntnis und Moralität, woran der Transparenzbegriff nun anschließt.

Die Forderungen nach Transparenz gehen mit dem Medienwandel und seinen gesellschaftlichen Auswirkungen einher. Max-Otto Baumann bezeichnet als eigentliche Triebfeder für mehr Transparenz das Internet: Dieses setze derartige Informationsmengen frei, dass Grenzen von Raum und Zeit irrelevant erscheinen und eine totale Sichtbarkeit möglich werde. Forderungen nach umfänglicher Transparenz folgen daher den Charakteristika digitaler und vernetzter Technologien, so als könne mithilfe des Internets und digitaler Medien eine Vision besserer Politik realisiert werden, welche im Prinzip seit der Antike bestehe. ${ }^{21}$

Transparenz tendiere jedoch dazu, zum systemischen Zwang zu werden, wie Byung-Chul Han schreibt: „Wer die Transparenz allein auf Korruption und Informationsfreiheit bezieht, verkennt ihre Tragweite. Die Transparenz ist ein systemischer Zwang, der alle gesellschaftlichen Vorgänge erfasst und sie einer tiefgreifenden Veränderung unterwirft."22

Die Einzelnen werden sichtbar und digital nachverfolgbar; dabei wirkt Transparenz sowohl faktisch als auch normativ. Faktisch, weil durch die Digitalisierung Nachverfolgbarkeit ermöglicht wird; normativ, weil die Datenabgabe zumeist freiwillig passiert und sich zu einem eingeforderten Standard und zur Norm entwickelt - so wird das Individuum einem entsprechenden Transparenzzwang unterworfen. Transparenz bezeichnet sodann nicht nur eine demokratische Grundsatzforderung zur Offenlegung staatlicher Geheimhaltung, sondern bezieht sich auf die Gesellschaft als ganze, was diese grundlegend tangiert.

Transparenz, verstanden als digitale Nachverfolgbarkeit des Individuums, kann dabei durchaus als Gegensatz zur Anonymität verstanden werden, die digitaler Kommunikation ebenso inhärent ist. ${ }^{23}$

20 Vgl. z.B. ebd. (S. 13).

21 Vgl. Baumann (2014: S. 398 f.); vgl. auch Hagendorff (2017: Kap. 11).

22 Han (2013: S. 6).

23 Zum komplexen Antagonismus von Anonymität und Transparenz vgl. auch Helm (2017). Paula Helm begreift beide Phänomene als Mechanismen zur Korrektur von diskursiven Ungleichheiten. 


\section{Ebenen und Dimensionen von Anonymität und Transparenz}

Nachdem Thiel die oben erörterten beiden Pole möglicher Konsequenzen von Anonymität im Netz unterschieden hat, unterscheidet er zudem zwei Bezugsebenen von Anonymität: eine horizontale und eine vertikale Ebene. ${ }^{24}$ Auf horizontaler Ebene anonym sei man unter Gleichgestellten, beispielsweise unter miteinander Kommunizierenden. Im digitalen Raum ist horizontale Anonymität gegeben, wenn unter einem Pseudonym gepostet wird und damit der Bezug zur wahren Identität, etwa dem Klarnamen einer Person, nicht gegeben ist. Vertikale Anonymität hingegen beziehe sich auf ein Macht- bzw. Hierarchieverhältnis, also auf die Identifizierbarkeit und Nachverfolgbarkeit eines Individuums für eine übergeordnete Instanz. Eine übergeordnete Instanz ist zuerst einmal der Staat, doch kommen im Digitalen neue Akteure hinzu, etwa die Betreibenden von Plattform- und Kommunikationsdiensten, Internetanbieter etc. Im digitalen Raum auf vertikaler Ebene anonym zu bleiben erfordert nicht unerhebliches Wissen um technische Vorkehrungen, wie etwa die Nutzung spezieller Software zur Verschlüsselung. So „zeigt sich, dass in digitaler Kommunikation gleichzeitig die Anonymität gegenüber den Kommunikationspartnern zunehmen und gegenüber Dritten abnehmen kann."25

Das digitale Gegenüber weiß also unter Umständen nicht, wer sich am anderen Ende des Kommunikationskanals befindet; staatliche Stellen und privatwirtschaftliche Akteure wie Plattformbetreiber und dergleichen hingegen schon. Hier wird die politische Dimension von Anonymität deutlich, da die verschiedenen Dimensionen von Anonymität an Machtverhältnissen entlang verlaufen können.

In Anlehnung an Thiel kann entsprechend auch zwischen ,horizontaler und ,vertikaler' Transparenz des Individuums unterschieden werden. Vertikale Transparenz der Einzelnen entsteht gegenüber dem Staat oder auch Unternehmen, die dadurch eine demokratietheoretisch relevante Rolle erhalten; sie kann eine Gefahr für die (politische) Freiheit darstellen. ${ }^{26}$

24 Vgl. Thiel (2017).

25 Matzner (2016: S. 248).

26 Digitale Dienste bieten i.d.R. die Möglichkeit, von sich selbst im Internet veröffentlichte Informationen anderen Nutzenden zugänglich zu machen und dabei zwischen verschiedenen Gruppen und Niveaus zu unterscheiden. Diese Funktion wird dabei gelegentlich als ,Privatsphäreeinstellungen“ benannt. Die Dienstanbietenden sammeln derlei Informationen jedoch trotzdem im für die Nutzenden unsichtbaren Hintergrund der Anwendung, dem sogenannten Backend, und nutzen diese i.d.R. auch. Gleichwohl erscheinen sie auf der für die Nutzenden einsehba- 
Der freiheitseinschränkende und demokratiegefährdende Zusammenhang von potentieller Beobachtung durch übergeordnete Instanzen wie dem Staat und daraus resultierendem, selbstzensierendem Verhalten wird sowohl von philosophischer ${ }^{27}$ wie auch juristischer ${ }^{28}$ Seite ausgiebig diskutiert. Im Zusammenhang mit digitalen Kulturen stellt die Ansammlung, Auswertung und Weiterleitung von Daten durch Unternehmen eine neue Herausforderung dar, da die Nutzenden transparent gegenüber den Unternehmen sind und letztere Zugriff auf die Daten haben, wobei teils unklar bleibt, was genau mit diesen Daten geschieht und welche Daten überhaupt anfallen; die Unternehmen selbst jedoch bleiben weitgehend intransparent gegenüber den Nutzenden:

Die beschriebene Intransparenz [der Unternehmen gegenüber den Nutzenden; Anm. L.W.] ist jedoch einseitig, schließlich beschreibt sie lediglich das Verhältnis der Endnutzer digitaler Medien zu den Wirtschaftsakteuren der IT-Branche, nicht aber das umgekehrte Verhältnis, welches freilich ein Verhältnis ist, welches sich durch hohe Transparenzgrade auszeichnet. [...] Während für die einen die Intransparenz der Datenverarbeitung und -verbreitung zum Auslöser des Kontrollverlusts wird, sind es für die anderen beispielsweise Leaks, mangelnde IT-Sicherheit oder Hackerangriffe, welche informationelle Kontrollverlustereignisse auslösen. ${ }^{29}$

Transparenz gegenüber den Anderen, Gleichgestellten entsteht etwa in sozialen Netzwerken zwischen Nutzenden: Als horizontale Transparenz kann entsprechend bezeichnet werden, dass durch individuelle Datenpreisgabe die Einzelnen nicht nur für Staat und Unternehmen transparent wer-

ren Anwendungsoberfläche, dem Frontend, je nach gewählter Reichweite teilweise nicht. Daraus ergibt sich ein Ungleichgewicht zwischen Anbietern und Nutzenden in Bezug auf die Einsicht in laufende Datenverarbeitungsprozesse: insofern kann in diesem Zusammenhang von einer vertikalen Transparenz der Einzelnen gesprochen werden. Die Einstellungen auf Ebene der Benutzeroberfläche schützen in diesem Sinne nicht vor der Kenntnisnahme durch die Plattformbetreiber und gegebenenfalls deren Werbepartner oder sogar einem potentiellen Missbrauch der Daten. Eine strikte Handhabung der (eigenen) sogenannten Privatsphäreeinstellungen mag also vor der Einsichtnahme durch unbekannte Nutzende - d. h. auf horizontaler Ebene - vor Transparenz schützen, nicht jedoch auf vertikaler Ebene. Vgl. Sobala/Watzinger (2019: S. 5).

27 Vgl. Rössler (2011); Foucault (1976).

28 Vgl. BVerfG, Urteil vom 15. Dezember 1983, Az. 1 BvR 209, 269, 362, 420, 440, 484/83; vgl. Simitis, NJW 1984, 398.

29 Hagendorff (2017: S. 191). 
den, sondern auch andere Personen realen oder potentiellen Zugriff auf diese Daten erhalten. ${ }^{30}$ Auf der vertikalen Ebene wiederum ist man im Digitalen - im Gegensatz zur analogen Welt - wesentlich einfacher und unbemerkt identifizierbar, da mit jeder Kommunikation und jedem Verhalten Datenspuren hinterlassen werden. Durch die kontinuierliche Generierung und Ansammlung von persönlichen wie auch Meta-Daten und deren dauerhafter Verfügbarkeit verflüchtigen sich digitale Interaktionen nicht in demselben Maße wie analoge, sondern bleiben auf unbestimmte Zeit nachvollziehbar, was Vorgänge auch im Nachhinein noch transparent macht und das Potential einer rückwirkenden Aufdeckung birgt. Auf diese Weise kann sich durch eine spätere Verknüpfung oder Auswertung von Daten eine auf horizontaler Ebene bestandene Anonymität auflösen und vormals anonyme Daten können einer Identität zugeordnet werden. ${ }^{31}$

Beide Dimensionen individueller Transparenz sind von zentraler Bedeutung, wobei auch sie unterschiedliche Auswirkungen zeitigen. Horizontale Transparenz lässt eine Entwicklung hin zu einer Gesellschaft der gegenseitigen Kontrolle als bedrohliches Szenario erscheinen; ${ }^{32}$ vertikale Transparenz hingegen birgt das Potential eines undemokratischen, totalitären Überwachungsregimes.

Die Spannung zwischen Nachvollziehbarkeit, Transparenz und Anonymität stellt, wie gezeigt werden konnte, ein zentrales Merkmal digitaler Kommunikation dar, woraus sich, wie Barbara Zehnpfennig bemerkt, „unmittelbar ein Spannungsverhältnis zur Frage der Verantwortung [ergibt; Anm. L.W.]. Denn Verantwortung impliziert Zurechenbarkeit.“"33 Zehnpfennig weist darauf hin, dass die Identifizierbarkeit von Personen die Übernahme von Verantwortung ermögliche, was eine ethische Grundlage für gesellschaftliches Miteinander sei. Hierfür stellt es ein Problem dar, wenn keine Person als Täter von Handlungen identifiziert werden kann: Motive und Persönlichkeit der Handelnden bleiben unbekannt, die han-

30 Horizontale Transparenz wird begünstigt durch die Freiwilligkeit der Datenpreisgabe. In ihrem Zusammenhang stellt sich die Frage, inwieweit Privatheit und Privatsphäreschutz im digitalen Zeitalter noch angemessen und zeitgemäß sind. Forderungen nach einer Klarnamenpflicht im Netz können dabei als Versuch, horizontale Transparenz herzustellen, verstanden werden. Vgl. z. B. Caspar, ZRP 2015, 233.

31 Vgl. Matzner (2016: S. 249).

32 Dies wird z. B. in Dave Eggers dystopischen Roman „The Circle“ fiktional erzählt, vgl. Eggers (2013).

33 Zehnpfennig (2017: S. 709). 
delnde Person kann auch nicht mit anderen Taten in Verbindung gebracht und daher insgesamt nicht zur Verantwortung gezogen werden.

\section{Philosophischer Brückenschlag: Hannah Arendts Überlegungen zur Selbstoffenbarung im Handeln und Sprechen}

Einen philosophischen Beitrag zum oben skizzierten Spannungsfeld leistet Hannah Arendt (1906-1975), die in ihrem Buch Vita activa Fragen (horizontaler) Anonymität und interpersonaler Sichtbarkeit im Handeln thematisiert.

Ihre Überlegungen auf ein Problem zu beziehen, das sich im Zuge der Digitalisierung stellt - die weit nach ihrer Schaffenszeit die Gesellschaft prägt - erweist sich insofern als gewinnbringendes ethisch-philosophisches Unterfangen, als Arendt die grundlegenden Bedingungen menschlichen Miteinanders thematisiert, welche auch und gerade in digitalen Kulturen zu Tage treten und unter Schlagworten wie Anonymität und Transparenz von Individuen in der Kommunikation verhandelt werden. Ihr Denken ist also geeignet für eine Anwendung auf digital geprägte Handlungskontexte, bei der zu bedenken bleibt, dass Arendts Überlegungen auf einer abstrakten Ebene Fragestellungen und Phänomene verbinden, die durch Digitalisierung und Vernetzung neue Relevanz erfahren, ohne jedoch konkrete Antworten auf die daraus resultierenden Problemstellungen zu geben.

\subsection{Sichtbarkeit im Handeln}

Arendt stellt in ihrer politischen Theorie die gegenseitige Sichtbarkeit der Einzelnen in den Vordergrund. Gemeinsam zu handeln ${ }^{34}$ stellt für sie den Kern menschlicher Freiheit dar, wobei es zentral sei, sich dabei zu zeigen und sichtbar - d. h. als Mensch und Person erkennbar - zu sein. (Horizontale) Anonymität bedeutet für Arendt hingegen, die Persönlichkeit der anderen nicht zu kennen, was Kommunikation in ihren Augen sinnlos macht.

Nach Arendts Interpretation können sich Menschen, wenn sie miteinander interagieren, nicht verstecken, sondern offenbaren sich stets: Perso-

34 Zur Einführung in die begriffliche Konzeption von Arendt vgl. z. B. Mahrdt (2011). 
nen enthüllen sich im Handeln und im Sprechen, hier stelle sich ihre Individualität dar, in der sie sich voneinander unterscheiden, anstatt bloß unterschiedlich zu sein. ${ }^{35}$ Durch sein Sprechen teile der Mensch also sich selbst mit, er kommuniziere nicht nur die Sache, über die er spricht, sondern auch seine Identität und Persönlichkeit, seine Menschlichkeit. Form und Inhalt laufen gewissermaßen parallel und es kann nicht eines ohne das andere sein:

Erst durch das gesprochene Wort fügt sich die Tat in einen Bedeutungszusammenhang, weil das Wort den Täter identifiziert und verkündet, dass er es ist, der handelt, nämlich jemand, der sich auf andere Taten und Entschlüsse berufen kann und sagen, was er weiter zu tun beabsichtigt. ${ }^{36}$

Durch die Einbettung von Handlungen in ein Miteinander durch Sprache werden diese einander zugeordnet. So entstehe das ,Bezugsgewebe menschlicher Angelegenheiten ${ }^{37}$, in dem Menschen sich einander unverkennbar zeigen: „Handelnd und sprechend offenbaren die Menschen jeweils, wer sie sind, zeigen aktiv die personale Einzigartigkeit ihres Wesens, treten gleichsam auf die Bühne der Welt, auf der sie vorher so nicht sichtbar waren $[\ldots]$..“38

Im Handeln und Sprechen komme folglich die individuelle Persönlichkeit zum Vorschein. Es werde deutlich, wer jemand sei. Vorzugeben, jemand anders zu sein, ist somit laut Arendt unmöglich, da im Gegensatz dazu, was einer sei, also welche Eigenschaften oder Merkmale jemand habe, „das eigentlich personale Wer-jemand-jeweilig-ist unserer Kontrolle darum entzogen [ist; Anm. L.W.], weil es sich unwillkürlich in allem mitoffenbart, was wir sagen oder tun. “39

Handeln, für das keine urhebende Person namhaft gemacht werden kann und das in der Anonymität verbleibt, sei daher sinnlos. Dabei begreift Arendt Anonymität als die Absenz von Persönlichkeit und Individualität und bezieht sie nicht lediglich auf den Namen von Personen. ${ }^{40}$ Anonymität in einem solchen Sinne einer Nicht-Erkennbarkeit stellt für Arendt entsprechend ein Problem für das menschliche Miteinander dar, da Handeln in der Anonymität keine Aufrichtigkeit zulasse. Die Möglich-

35 Vgl. Arendt (2002: S. 214).

36 Ebd. (S. 218).

37 Vgl. ebd. (Kapitel 5).

38 Ebd. (S. 219).

39 Ebd.

40 Vgl. Arendt (2002). 
keit, in der Masse aufzugehen und im Anonymen zu handeln, sieht sie entsprechend kritisch. Da Menschen einerseits gleich und andererseits verschieden seien, müssten sie, um miteinander auszukommen, politisch handeln. Für Arendt macht dies den Kern menschlichen Daseins aus. Als Menschen gleich und als Individuen verschieden zu sein, stellt somit die gesellschaftliche Anschlussfähigkeit und eine Einbettung in soziale Kontexte her, was für Arendt zwei Konsequenzen zeitigt: Einerseits ermöglicht es eine Kontinuität von Verständigung zwischen Individuen sowie über Zivilisationen und Epochen hinweg. Andererseits macht es differenzierten Austausch und komplexe Sprache notwendig, da jeder und jede Einzelne unterschiedlich ist; daher ist die Erkennbarkeit einer Person im Handeln von zentraler Bedeutung.

Es geht Arendt also um die Sichtbarkeit und Erkennbarkeit der Sprechenden und Handelnden im politischen Raum, in einem ganz wörtlichen Sinne einer physischen Präsenz - man steht sich von Angesicht zu Angesicht gegenüber - wie auch in einem übertragenen Sinne einer Identifizierbarkeit der individuellen Persönlichkeit.

\subsection{Mit Arendt digitale Kulturen verstehen?}

Wie sind nun solche an Arendt orientierten Überlegungen auf digitale Kulturen zu beziehen? Obwohl Arendt keine Medienphilosophin ist und sich mit Fragen des digitalen Wandels nicht beschäftigen konnte, sind ihre Überlegungen doch von erstaunlicher Aktualität. Phänomene von Anonymität und Transparenz in digitalen Kulturen sind vor diesem Hintergrund ambivalent zu bewerten.

Zum einen stellt sich die Frage, ob sich Menschen auch im Netz kommunikativ offenbaren, oder ob das Fehlen der physischen Präsenz das Wesenhafte eines Menschen zu verdecken vermag, also ob das Netz aus einer Arendt'schen Perspektive einen Ort der Anonymität darstellt oder nicht. Dabei lässt sich beobachten, dass auch im Internet, wo unter Pseudonym geschrieben werden kann und Identitäten scheinbar verschleiert werden können, sich das Individuum trotzdem zeigt, mit dem, was es wie schreibt und was es ausspart. Dies zeigt Inga Tappe detailliert: ${ }^{41}$ Sie argumentiert mit Arendt dafür, dass sich Menschen mit ihrer Persönlichkeit auch im Internet zeigen und somit auch im digitalen Raum deutlich werde, wer je-

41 Vgl. Tappe (2015). 
mand sei. ${ }^{42}$ Denn im Netz könne sich jeder zeigen, da es zumindest einen gewissen Zugang zur Bühne der Öffentlichkeit biete. Gleichzeitig ermöglicht das Internet eine Vernetzung mit Anderen und stellt eine Art digitales Bezugsgewebe der menschlichen Angelegenheiten dar. Durch den Austausch von Informationen darüber, was einer ist, lasse sich eben auch in der digitalen Kommunikation ein Rückschluss darüber ziehen, wer einer ist. Im digitalen Raum können sich nach Tappes Interpretation Menschen kommunizierend offenbaren - hier zeigt sich ein (im Arendt'schen Sinne) politisches Potential des Internets, welches wahrhaftige Kommunikation ermöglicht und ohne persönliche und physische Präsenz globalisiert. Das Internet stellt sich aus Arendt'scher Perspektive demnach durchaus als ein Ort der Pluralität und des Handelns dar.

Zum anderen gilt es die zweite Tendenz des Digitalen, die Transparenz der Einzelnen, mit Arendts Denken in Verbindung zu bringen. Julia Maria Mönig fragt hier, welchen Erkenntniswert digitale Datenaufzeichnungen und deren Auswertung überhaupt generieren und ob das Wesenhafte einer Person, das wer-einer-ist, anhand von Daten erfasst werden könne. ${ }^{43}$ In jedem Falle entstehe ein kommunikatives Bild von einer Person - was Mönig dafür argumentieren lässt, dass auch Datenprofile o. ä. das Wesenhafte eines Individuums zu offenbaren ermöglichten. Dies sei die

[...] Dimension, die das massenhafte Datensammeln so sensibel macht, auch von sogenannten Metadaten. Es kann eben nicht nur rekonstruiert werden, was einer ist, sondern durch seine Verbindungen $\mathrm{zu}$ anderen Menschen wird auch das ihm eigentlich Wesenhafte eingefangen. ${ }^{44}$

Durch das Sammeln von Informationen über eine Person könne nicht nur nachvollzogen werden, was einer ist, sondern durch soziale Netzwerke werden Aufzeichnungen über soziale Beziehungen in großem Umfang und mit relativ geringem Aufwand möglich, was auch einen Eindruck vermittle, wer diese Person sei. Mönig zufolge ist die gegenwärtige gesamtgesellschaftliche Ausweitung von Überwachungstendenzen nicht zuletzt aus einer an Arendt orientierten Perspektive äußerst kritisch zu sehen. Folgt man einer solchen Sichtweise, stellt sich die Transparenz, die digitaler Kommunikation inhärent ist, als eine Gefahr für die Freiheit und Wahr-

42 Dies ist erst einmal unabhängig vom Namen einer Person oder ihrer juristischen Identifizierbarkeit der Fall.

43 Vgl. Mönig (2015: S. 103).

44 Ebd. (S. 105). 
haftigkeit kommunikativen Miteinanders dar. Die Transparenz des Individuums, die durch Datafizierung entsteht, unterscheidet sich nämlich von der Sichtbarkeit und Offenbarung im Sprechen, von der Arendt schreibt und die sie für elementar hält. Das aus digitalen Medien für das Individuum entstehende Überwachungspotential könne Freiheit und Selbstbestimmung erheblich einschränken: Überwachung ist für Arendt ein Merkmal totalitärer Herrschaft. 45

Für die Reflexion des Zusammenhangs von Anonymität, Transparenz und Verantwortung in digitalen Kulturen wurden diese drei Begriffe hier in eine kritische Beziehung zueinander gesetzt. Arendts Philosophie folgend wurde sodann konturiert, welche Rolle Anonymität und Sichtbarkeit in digitalen Kulturen spielen. Aus ihrem Verständnis heraus ist es zentral, dass Menschen, wenn sie politisch handeln, füreinander erkennbar sind. Ihre Unterscheidung des was und des wer jemand ist, lässt sich interpretieren als ein Plädoyer für zwischenmenschliche Wahrhaftigkeit, die durch Identifizierbarkeit entsteht: Menschen können gar nicht anders, als sich im Handeln zu zeigen, die Art und Weise wie jemand handelt, erhellt bzw. entblößt das wer. Dieser Zusammenhang zeige sich, so Tappe, auch im Internet, das damit durchaus einen Raum sozialer Erscheinungen biete. Dabei ist mit Arendt wichtig zu betonen, dass sich die Menschen im Handeln zeigen und identifizierbar machen, aber nicht transparent. Ein Abgleiten in die Anonymität und ein Verlust individueller Merkmale verunmöglicht demnach politische Verständigung. Arendt schwebt keine Gesellschaft transparenter Individuen vor, in der alle alles über die anderen wissen; für sie zählt die Gewissheit, wer das Gegenüber ist, im Sinne seiner Persönlichkeit, nicht seines Namens.

\section{Fazit}

Um die Phänomene des Internets philosophisch einordnen zu können, gilt es, vorhandene Theorien und Begrifflichkeiten auf die veränderten Phänomene und Umstände zu beziehen: ${ }^{46}$ Dazu wurden die grundlegenden Überlegungen zum Menschen in einer sich verändernden technisierten Welt von Hannah Arendt herangezogen, da anhand dieser auch im digitalen Kontext bestehende Fragen thematisiert werden konnten. Zwar zeichnet Arendt selbst ein eher pessimistisches Modell der ihr zeitgenössischen

45 Vgl. Mönig (2015).

46 Vgl. Thies (2018: S. 137). 
Gesellschaft, gleichzeitig zeigt sie jedoch auf, dass Gesellschaft stets menschengemacht und damit veränderbar ist.

In diesem Beitrag wurde daher eine Verbindung hergestellt zwischen Foucaults Theorie der Autorschaft, die dazu diente, das Phänomen der Anonymität zu verstehen, Thiels Überlegungen zur Anonymität in digitalen Kulturen sowie Arendts Philosophie des Handelns, die eine Offenbarung des Individuums voraussetzt. Reflektiert wurde dabei die Frage, was Anonymität und Transparenz in digitalen Kulturen für die Verantwortlichkeit von Menschen bedeuten können. Dabei wurde deutlich, dass die $\mathrm{Zu}$ ordnung von Verantwortung und Autorschaft auch im Internet zentral bleibt. Arendt plädiert für persönliche Sichtbarkeit im Handeln und Kommunizieren, jedoch gegen die Überwachung freier Individuen und ist daher auf digitale Kontexte ambivalent zu beziehen, da im Internet der Antagonismus aus Anonymität und Transparenz ein ubiquitäres Phänomen darstellt.

\section{Literaturverzeichnis}

Arendt, Hannah (2002): Vita activa oder Vom tätigen Leben. München: Piper.

Baumann, Max-Otto (2014): „Die schöne Transparenz-Norm und das Biest des Politischen: Paradoxe Folgen einer neuen Ideologie der Öffentlichkeit“. In: Leviathan 42 (3), S. 398-419.

Caspar, Johannes (2015): „Klarnamenpflicht versus Recht auf pseudonyme Nutzung“. In: Zeitschrift für Rechtspolitik (ZRP) 48 (8), S. 233-236.

Digitales Wörterbuch der deutschen Sprache (o. J.): transparent. URL: https:// www.dwds.de/wb/transparent [Abruf am: 07.10.2020].

Eggers, Dave (2013): The Circle. New York: Penguin Books.

Foucault, Michel (1976): Überwachen und Strafen. Die Geburt des Gefängnisses. Frankfurt am Main: Suhrkamp.

Foucault, Michel (2000): „Was ist ein Autor?“. In: Jannidis, Fotis et al. (Hrsg.): Texte zur Theorie der Autorschaft. Stuttgart: Reclam, S. 198-229.

Hagendorff, Thilo (2017): Das Ende der Informationskontrolle. Digitale Mediennutzung jenseits von Privatheit und Datenschutz. Bielefeld: transcript.

Han, Byung-Chul (2013): Transparenzgesellschaft. Berlin: Matthes \& Seitz.

Härting, Niko (2013): „Anonymität und Pseudonymität im Datenschutzrecht“. In: Neue Juristische Wochenschrift (NJW) 66 (29), S. 2065-2071.

Helm, Paula (2017): „Transparenz und Anonymität: Potentiale, Grenzen, Irrtümer“. In: Forschungsjournal Soziale Bewegungen 30 (2), S. 142-151.

Lück, Anne-Kathrin (2013): Der gläserne Mensch im Internet. Ethische Reflexionen zur Sichtbarkeit, Leiblichkeit und Personalität in der Online-Kommunikation. Stuttgart: Kohlhammer. 
Mahrdt, Helgard (2011): „Arbeiten/Herstellen/Handeln“. In: Heuer, Wolfgang et al. (Hrsg.): Arendt-Handbuch. Leben - Werk - Wirkung. Stuttgart: Metzler, S. 265-268.

Matzner, Tobias (2016): „Anonymität“. In: Heesen, Jessica (Hrsg.): Handbuch Medien- und Informationsethik. Stuttgart: Metzler, S. 248-254.

Menéndez-Viso, Armando (2009): „Black and white transparency: contradictions of a moral Metaphor“. In: Ethics and Information Technology 11 (2), S. 155-162.

Mey, Stefan (2018): Darknet. Waffen, Drogen, Whistleblower. Wie die digitale Unterwelt funktioniert. München: C.H. Beck.

Mönig, Julia Maria (2015): Vom »oikos« zum Cyberspace. Bielefeld: transcript.

Rössler, Beate (2001): Der Wert des Privaten. Frankfurt am Main: Suhrkamp.

Simitis, Spiros (1984): „Die informationelle Selbstbestimmung - Grundbedingung einer verfassungskonformen Informationsordnung“. In: Neue Juristische Wochenschrift (NJW) 37 (8), S. 398-405.

Sobala, Felix/Watzinger, Lea (2019): „Alternative Internetanwendungen und was sie anders machen. Über Geschäftsmodelle, Datenverarbeitungsebenen und gesellschaftliche Aspekte“. In: Privatheit und Digitalisierung 12, S.3-10. URL: https://issuu.com/grkprivatheitdigitalisierung/docs/magazin_12_dezember_20 19_[Abruf am: 06.10.2020].

Tappe, Inga (2015): „Was einer ist und wer einer ist. Anonymität und Identität in Sozialen Medien aus philosophischer Sicht“. In: Grimm, Petra et al. (Hrsg.): Anonymität und Transparenz in der digitalen Gesellschaft. Stuttgart: Franz Steiner, S. 77-95.

Thiel, Thorsten (2016): „Anonymität und der digitale Strukturwandel der Öffentlichkeit“. In: Zeitschrift für Menschenrechte 10 (1), S. 9-24.

Thiel, Thorsten (2017): „Anonymität und Demokratie“. In: Forschungsjournal Soziale Bewegungen 30 (2), S. 152-161.

Thies, Christian (2018): „Verantwortung im digitalen Weltsystem. Grundsätzliche Überlegungen zu einem neuen Bereich angewandter Ethik“. In: Burk, Steffen et al. (Hrsg.): Privatheit in der digitalen Gesellschaft. Berlin: Duncker \& Humblot, S. 137-152.

Wegener, Bernhard (2006): Der geheime Staat. Arkantradition und Informationsfreiheitsrecht. Göttingen: Morango.

Weidacher, Georg (2019): „Die Ideologie der Transparenz. Werte- und Bewertungskonflikte im Diskurs über Transparenz, gläserne Menschen und Überwachung vor dem Hintergrund internet-geprägter Lebensformen“. In: Stehen, Pamela/ Liedtke, Frank (Hrsg.): Diskurs der Daten. Berlin und Boston: De Gruyter, S. 97-122.

Zehnpfennig, Barbara (2017): „Verantwortung in den Medien“. In: Heidbrink, Ludger (Hrsg.): Handbuch Verantwortung. Wiesbaden: Springer VS, S. 697714. 
